\title{
Postnatal changes of interleukin-18 receptor immunoreactivity in neurons of the retrosplenial cortex in wild-type and interleukin-18 knock out mice
}

\author{
By
Tetsu HAYAKAWA ${ }^{1}$, Masaki HATA ${ }^{1}$, Sachi KUWAHARA-OTANI ${ }^{2}$, Hideshi YAGI ${ }^{2}$, Haruki OKAMURA ${ }^{1}$ \\ ${ }^{1}$ Laboratory of Tumor Immunology and Cell Therapy Hyogo College of Medicine Mukogawa, \\ Nishinomiya, Hyogo 663-8501, Japan \\ ${ }^{2}$ Department of Anatomy Hyogo College of Medicine Mukogawa, Nishinomiya, Hyogo 663-8501, Japan
}

- Received for Publication, August 17, 2017-

\begin{abstract}
Key Words: immunohistochemistry, cytokines, development, IL-18 receptor, laminar distribution, cerebral cortex
Summary: Interleukin-18 (IL-18), which is involved in the inflammatory response, is also found in the cerebral cortex. IL-18 receptor-immunoreactive (IL-18R-ir) neurons are present in layer $\mathrm{V}$ of the retrosplenial cortex (RSC). In the adult IL-18 knock out (KO) mice, no IL-18R-ir neurons but many degenerated neurons are present in layer V of the RSC, suggesting that any changes in the neurons of layer $V$ have occurred during postnatal development. We examined changes of IL-18R expression during postnatal development. In the wild-type mice, many IL-18R-ir neurons were present in layers II, III and VI of the RSC in 2-week-old mice, whereas they were sparsely observed in only layer III in 3-week-old mice. No IL-18R-ir neurons were present in 4- and 5-week-old mice. In older than 6-week-old mice, many IL-18R-ir neurons were present in layers $\mathrm{V}$ and VI. The IL-18KO mice showed IL-18R-ir neurons in layers II, III and VI at 2-weeks-old, and a few in layer III at 3-week-old mice, similar to that in the wild-type mice. No IL-18R-ir neurons were found in mice older than 4 weeks of age. Thus, IL-18 or IL-18R seem to be involved in the construction of neural circuits corresponding to events after 3-weeks of age.
\end{abstract}

\section{Introduction}

Interleukin 18 (IL-18) was originally isolated as an interferon-gamma inducing factor from Kupffer cells of mice (Okamura et al., 1995). IL-18 is thought to be a proinflammatory cytokine in $\mathrm{T}$ lymphocytes and play proapoptotic, proatherogenic roles in several diseases (Dinarello and Fantuzzi, 2003; Reddy, 2004; Dinarello, 2006). IL-18 has also been found in nonimmune tissues, such as, the adrenal gland, the mammary gland, the pituitary gland, the renal tubule, the ependymal cells, the neurons in the medial habenula, Purkinje neurons in the cerebellum, and the glial cells of the dorsal root ganglion and the trigeminal spinal nucleus caudalis. (Conti et al., 2000; Takahata et al., 2001; Sugama et al., 2002; Nagai et al., 2005; Wang et al., 2006; Dinarello, 2007; Miyoshi et al., 2008; Daigo et al., 2012). IL-18 synthesis in the microglia is elevated by neuropathic pain (Miyoshi et al., 2008; Daigo et al., 2012). There are also neurons contain- ing the IL-18 receptor (R) in the central nervous system, such as in the retrosplenial cortex (RSC), hippocampus, hypothalamus, septum, supraoptic nucleus and Purkinje neurons in the cerebellum (Sugama et al., 2002; Andoh et al., 2008; Alboni et al., 2009; Hayakawa et al., 2016; Kuwahara-Otani et al., 2017).

Behavioral studies of the IL-18 knock out (KO) mice have shown that rearing activity is significantly suppressed, retention latency is much shorter, and acquisition latency is significantly prolonged in the water maze test for IL-18KO mice as compared with those for the wildtype mice (Yaguchi et al., 2010; Too et al., 2014). Lesion studies of different parts of the RSC in rats have shown that differential degrees of an impairment for alternations in spatial memory and motor activity (Van Hoesen et al., 1991; Wyss and Van Groen, 1992; Vann et al., 2003; Vann and Aggleton, 2004; Sipos et al., 2007; Pothuizen et al., 2008). These results suggested that the degeneration of the IL18R-ir neurons in the RSC may cause behavioral 
abnormalities for the IL-18KO mouse.

Our previous study (Hayakawa et al., 2016) has revealed that the adult IL-18KO mice showed no IL-18Rimmunoreactive (IL-18R-ir) neurons in the RSC, whereas many IL-18R-ir neurons are found specifically in layers $\mathrm{V}$ and VI of the adult wild-type mice. The IL-18R-ir neurons were not recognized in the RSC even in 5-weekold IL-18KO mice. Electron microscopic observation has shown that many small to medium-sized electron-dense neurons are present in layer $\mathrm{V}$ of the RSC in IL-18KO mice (Hayakawa et al., 2016). Thus, the IL-18R-ir neurons are thought to degenerate in IL-18KO mice that are older than 5 weeks. These results suggested that IL-18R-ir neurons may change their immunoreactivity or degenerate in IL-18KO mice younger than 5 weeks old. However, it is not clear when the IL-18R-ir neurons stop expressing immunoreactivity and when the IL-18R-ir neurons degenerate or why the IL-18R-ir neurons disappear in the RSC. Furthermore, there have not been any studies investing the change of immunoreactivity during postnatal development of IL-18R-ir neurons in the RSC of the wild-type and the IL-18KO mice.

In the present study, we investigated when IL-18Rir neurons or presumed IL-18-containing neurons in the $\mathrm{RSC}$, disappear in the IL-18KO mice. We also attempt to clarify if IL-18R immunoreactivity changes in neurons in the RSC during postnatal development from 2 weeks to 8 weeks old in wild type and IL-18KO mice. Because IL-18 and IL-18R are thought to be co-localize in the neurons of the brain (Andoh et al., 2008; Alboni et al., 2009; Kuwahara-Otani et al., 2017), we used the IL-18R immunoreactivity as a marker for IL-18-containing neurons.

\section{Materials and methods}

A total of 45 wild-type mice (C57BL/6, male) obtained from Japan SLC and IL-18 KO (IL-18 ${ }^{-1-}$, male) mice from 2 to 8 weeks of ages were used. IL- $18^{-/-}$mice gifted by Prof. S. Akira (Osaka Univ.) were backcrossed with C57BL/6 mice; F8 mice were used (Takeda et al., 1998). The number of mice used at each week of age is shown in Table 1. All procedures were approved by the Animal Care and Use Committee of Hyogo College of Medicine and were in compliance with the National Institutes of Health Guide for the Care and Use of Laboratory Animals.

To determine the distribution of IL-18R-ir neurons in the RSC, wild-type mice or IL-18KO mice at each week old of ages were anesthetized first with $2 \%$ isoflurane in
$30 \%$ oxygen and $70 \%$ nitrous oxide in a chamber, which they breathed spontaneously. A tube was then attached to the nose to administer isoflurane, and the anesthetized mouse was placed in a supine position. The mouse was perfused first with $5 \mathrm{ml}$ of saline and then with $30 \mathrm{ml}$ of a fixative containing $4 \%$ paraformaldehyde in $0.1 \mathrm{M}$ phosphate buffer at $\mathrm{pH}$ 7.4. The brain was immediately removed and placed in the same fixative for 1 day. Serial frontal sections were made at a thickness of $70 \mu \mathrm{m}$ using Microslicer $^{\mathrm{TM}}$ (DosakaEM, Kyoto). The sections were incubated with a polyclonal goat anti-IL-18R alpha serum (R\&D Systems, Minneapolis, MN; 1:200, AF856) in 0.1 $\mathrm{M}$ phosphate buffer at $\mathrm{pH} 7.4$ containing $0.3 \%$ Triton $\mathrm{X}-100$ for 1 day. The treated sections were incubated with biotinylated rabbit anti-goat IgG (Vector Laboratories, Burlingame, CA; 1:200) for $5 \mathrm{~h}$, then incubated with Vectastain ${ }^{\circledR} \mathrm{ABC}$ reagents (Vector Laboratories) for 1 day, and finally reacted with a solution of $0.1 \% 3,3$ '-diaminobenzidine tetrahydrochloride (DAB) and $0.01 \% \mathrm{H}_{2} \mathrm{O}_{2}$ in $0.05 \mathrm{M}$ phosphate buffer at $\mathrm{pH} 7.4$ for $5 \mathrm{~min}$ to produce brown reaction products. Control sections of both wild type and IL-18KO mice were proceeded the same with the exception of the primary antibody incubation.

\section{Results}

In 2-week-old wild type mice, many IL-18R-ir neurons were distributed in the RSC (Fig. 1). The IL-18R-ir neurons were present throughout the rostrocaudal extent of the RSC. At the caudal end of the RSC, the IL-18Rir neurons extended to the subiculum. Many IL-18R-ir neurons were found specifically in layers II and III, and fewer IL-18R-ir neurons were found in layer VI. The IL18R-ir neurons in layer II and III were small and round in shape, having small granular DAB reaction products (Fig. 1D), and were distributed only in the granular RSC (Figs. 1A-C). The IL-18R-ir neurons in layer VI were oval or triangular in shape (Fig. 1E), and were distributed mainly in the granular RSC, though some labeled neurons extended to the agranular RSC (Fig. 1A-C). No IL-18Rir fibers and terminals were found. In 3-week-old mice, the IL-18R-ir neurons had mostly disappeared, but a few IL-18R-ir neurons were found in layer III throughout the rostrocaudal extent of the RSC. At this age, the number of IL-18R-ir neurons was small and their immunoreactivity was low (Fig. 2A).

No IL-18R-ir neurons were present in 4- and 5-weekold mice in any layers of the RSC (Fig. 2B). In 6-week-

Table 1. The number of mice used at each week of age

\begin{tabular}{cccccccc}
\hline & 2 weeks & 3 weeks & 4 weeks & 5 weeks & 6 weeks & 7 weeks & 8 weeks \\
\hline Wild type & 3 & 3 & 3 & 3 & 3 & 2 & 6 \\
IL-18 KO & 3 & 3 & 3 & 3 & 2 & 2 & 3 \\
\hline
\end{tabular}



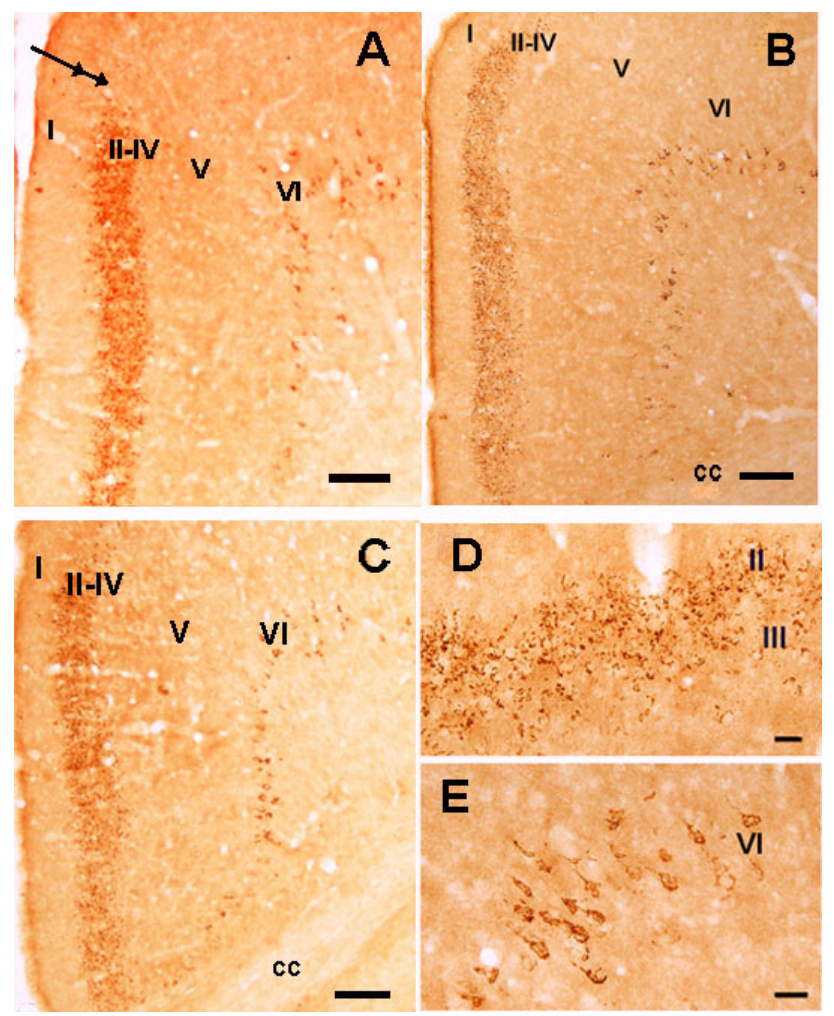

Fig. 1. Photomicrographs of immunohistochemistry for interleukin-18 receptor (IL-18R) in the RSC of 2-week-old wild type mice. Many IL-18R immunoreactive neurons are present in layer II, III and VI. (A) Rostral portion of the RSC. Double-arrow indicates the border between the granular and agranular cortex of the RSC. (B) Mid-portion of the RSC at the level of the habenula. (C) Caudal portion of the RSC. (D) High power photomicrograph of the IL-18R-immunoreactive neurons in layers II and III of the RSC. (E) High power photomicrograph of the IL-18R-immunoreactive neurons in layer VI of the RSC. CC, corpus callosum. Scale bars $=100 \mu \mathrm{m}$ in A, B, C and $20 \mu \mathrm{m}$ in D and E. old mice, many IL-18R-ir neurons were present in layers $\mathrm{V}$ and VI. The IL-18R-ir neurons in layer V were distributed mostly in the outer part of layer $\mathrm{V}$ and the granular RSC (Fig. 2C). The IL-18R-ir neurons in layer VI were distributed mainly in the granular RSC, but some extended to the agranular RSC. In 7- and 8-week-old mice, many IL-18R-ir neurons were found in layers V and VI of the RSC (Fig. 2D). The IL-18R-ir neurons were round or oval in shape, and were medium-sized neurons containing granular DAB reaction products. They were distributed specifically in the outer part of layer $\mathrm{V}$ and the granular RSC. The number of IL-18R-ir neurons was larger and their immunoreactivity was higher than those of 6-weekold mice (Fig. $2 \mathrm{C}$ and D). Many IL-18R-ir neurons were also found in layer VI. They were distributed mostly in the granular RSC but some IL-18R-ir neurons extended to the agranular RSC, similar to those of 6-week-old mice. No IL-18R-ir fibers and terminals were found. IL-18Rir neurons were also found in the subiculum, the medial septum and the diagonal band of Broca in all ages of the wild-type mice.

In 2-week-old IL-18KO mice, many IL-18R-ir neurons were found in the RSC (Fig. 3A), specifically in layers II and III, and were distributed in the granular RSC throughout the rostrocaudal extent. Many IL-18R-ir neurons were also found in layer VI, and were distributed mostly in the granular RSC, though some extended to the agranular RSC similar to the distribution in the wild type mice. At the caudal end, the labeled neurons extended to the subiculum (Fig. 3B). In 3-week-old mice, a few IL-18R-ir neurons were found in only layer III (Fig. 3C), and they were distributed throughout the rostrocaudal extent of the RSC. The number of IL-18R-ir neurons was small, and their immunoreactivity was low. IL-18R-ir neurons were found in the subiculum, the medial septum and the diagonal
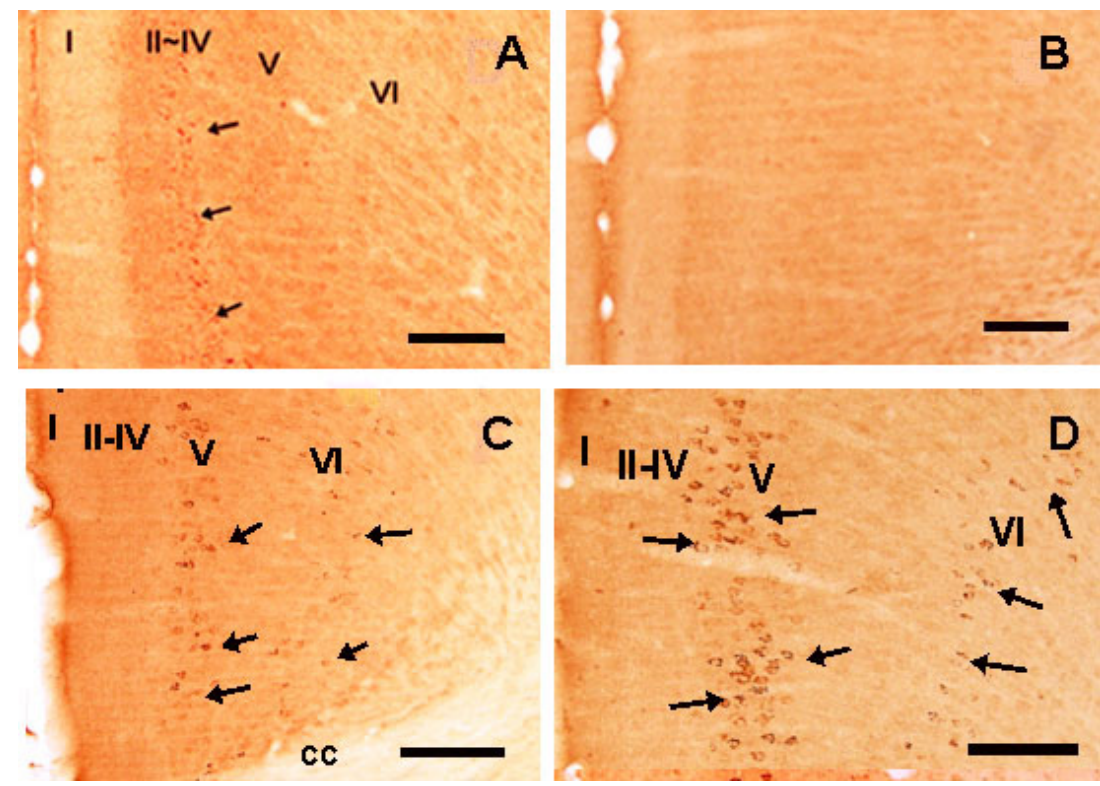

Fig. 2. Immunohistochemistry for IL-18R in the RSC of wild-type mice at 3-weekold (A), 4-week-old (B), 6-week-old (C) and 7-week-old (D) wild type mice. (A) Some IL-18R immunoreactive neurons (arrows) were found in layer III. (B) No IL-18R immunoreactive neurons were found in the RSC. (C) Several IL-18R immunoreactive neurons (arrows) were found in layers V and VI. (D) Many IL-18R immunoreactive neurons (arrows) were found in the outer part of layers $\mathrm{V}$ and VI. Scale bars $=100 \mu \mathrm{m}$. 


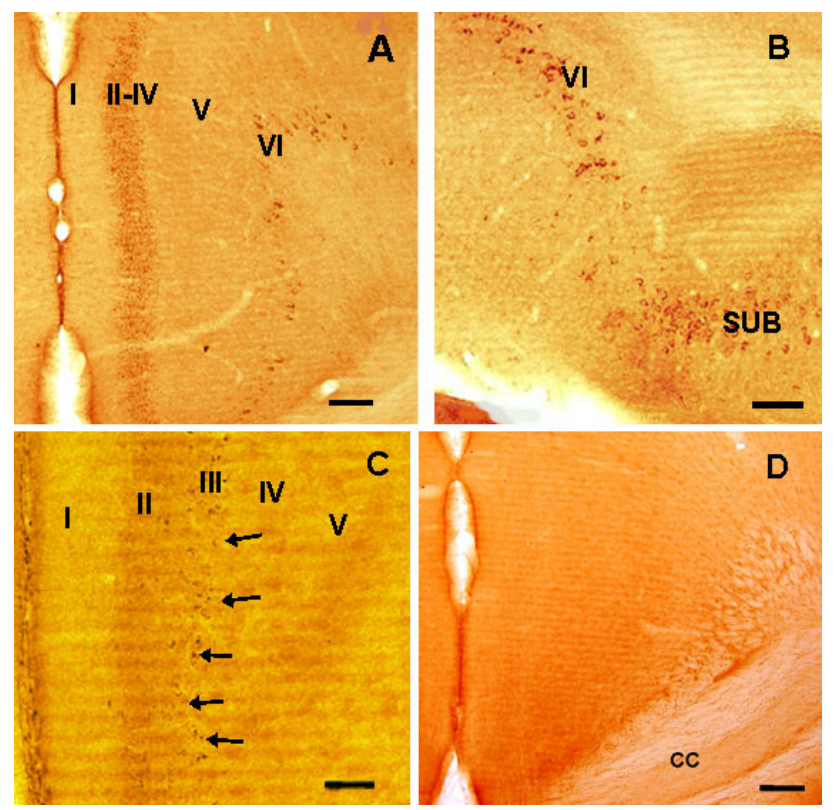

Fig. 3. Immunohistochemistry for IL-18R in the RSC of 2-week-old (A and B), 3-week-old (C), and 7-week-old (D) of the IL-18 knock out mice. (A) Many IL-18R immunoreactive neurons were present in layers II, III and VI at the rostral part of the RSC. (B) IL-18R-immunoreactive neurons in layer VI extended to the subiculum (SUB) at the caudal end of the RSC. (C) Some IL-18R immunoreactive neurons (arrows) were present in layer III. (D) No IL-18R-immunoreactive neurons were present in the RSC. CC, corpus callosum. Scale bars $=100 \mu \mathrm{m}$ in A, B and D, and $50 \mu \mathrm{m}$ in $\mathrm{C}$. band of Broca in the 2- and 3-week-old IL-18KO mice, similar to the distribution in the wild type mice. No IL18R-ir neurons were found in the RSC in either 4-weekold or 5-week-old IL-18KO mice, similar to the finding in wild type mice. Furthermore, IL-18KO mice older than 6 weeks of age showed no IL-18R-ir neurons in the RSC (Fig. 3D). IL-18R-ir neurons were found in the medial septum and the diagonal band of Broca, but not in the subiculum, in IL-18KO mice older than 6 weeks of age. No IL-18R-ir neurons were found in the control sections for either the wild type or IL-18KO mice. Within the group of each week of age, the distribution patterns of IL18R-ir neurons were almost same.

\section{Discussion}

The present results showed that many IL-18R-ir neurons were present specifically in layers II, III and VI at 2 weeks of age, whereas IL-18R-ir neurons disappeared from 4 to 5 weeks of age in the wild-type mice. Additionally, many IL-18R-ir neurons appeared in layers V and VI of the RSC in wild-type mice older than 6 weeks of age. Interestingly, many IL-18R-ir neurons were also identified specifically in layers II, III and VI in the IL-18KO mice. IL-18R-ir neurons were no longer present in the RSC of IL-18KO mice older than 4 weeks of age (Fig. 4).

Alboni et al. (2009) have reported the distribution of total IL-18R alpha mRNAs in adult wild-type mice determined by in situ hybridization, which were distributed in
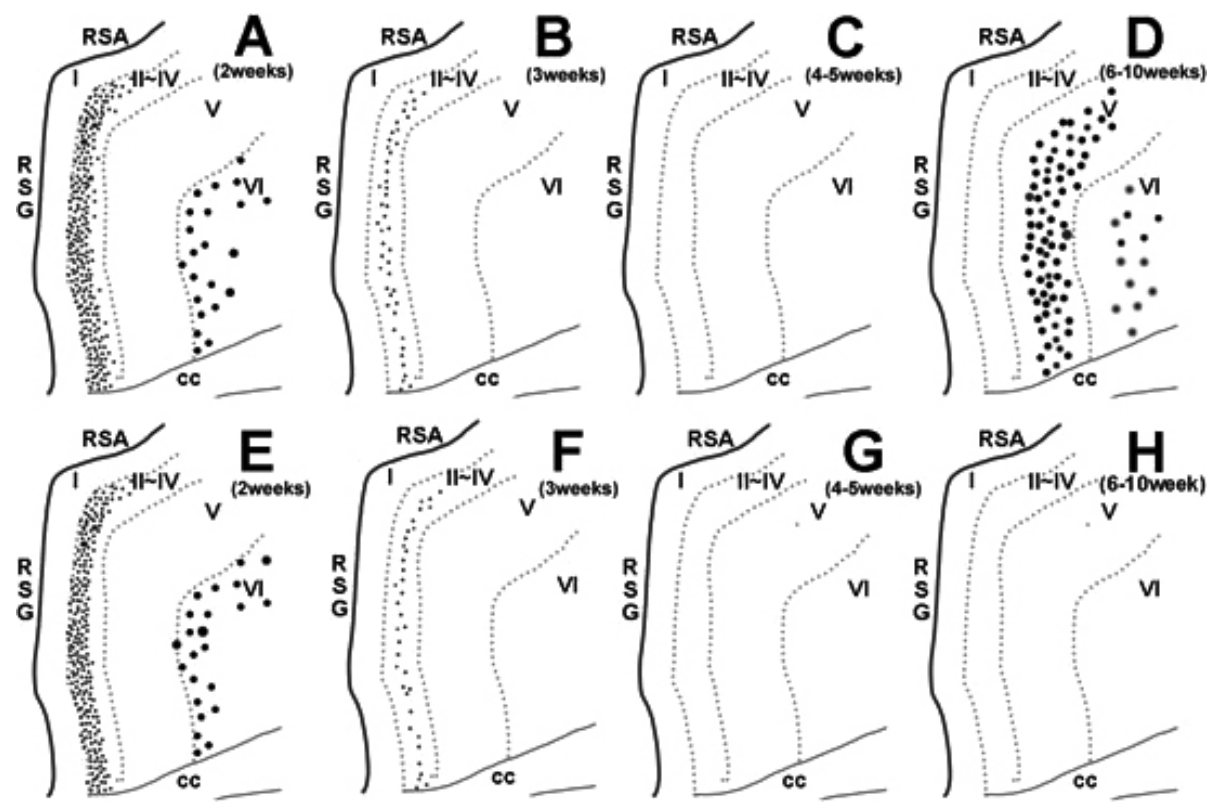

Fig. 4. Schematic drawings of changes in the distribution of IL-18R immunoreactive neurons in the RSC during postnatal development in the wild type (A-D) and the IL-18 knock out (E-H) mice. Black circles indicate IL-18R immunoreactive neurons. CC, corpus callosum; RSA, agranular retrosplenial cortex; RSG, granular retrosplenial cortex. 
neurons of the olfactory bulb, cerebral cortex including the RSC, hypothalamus, habenula, hippocampus, and the cerebellar cortex. The mRNAs for IL-18R alpha were present in all layers of the cortex, where type I IL-18R alpha was localized primarily in layers V and VI, and type II IL-18R alpha was mainly in layer V. Immunohistochemistry for IL-18R alpha revealed a similar distribution to that of the gene expressions determined by in situ hybridization. However, the authors did not describe the precise laminar distribution of IL-18R-ir neurons in the RSC. Our present results in adult wild-type mice agree with their reported distribution of IL-18R-ir neurons in the RSC, whereas the distribution in the 2-week old wildtype mice showed IL-18R-ir neurons specifically in layers II, III and VI but not in layer V.

During the early postnatal period, postmitotic precursor cells in the dorsomedial part of the neonatal ventricular zone migrate into the medial limbic cortex and the RSC during the first postnatal week. These neurons migrate and display morphological changes, and enter into layer II of the cortex until around 2 weeks of age. In the RSC, these cells give rise to dendritic bundles in layer I (Zgraggen et al., 2012). Parvalbumin and calbindin D28k-immunoreactive neurons are distributed in all layers of the RSC (Luth et al., 1993; Salaj et al., 2015). Changes in parvalbumin and calbindin D28k immunoreactivities have been reported in the RSC of rats during postnatal development (Alcantara et al., 1993). Parvalbumin-immunoreactive neurons first appear in layer $\mathrm{V}$ and later in layers VI and IV, and then in II and III at postnatal days 8 or 9 . Adult patterns are reached at the end of 3 weeks of age. Calbindin D28K-immunoreactive neurons are present at birth in all cortical layers except the molecular layer. Heavily labeled calbindin D28K-immunoreactive neurons decrease in number from postnatal days 11 to 15 , and adult patterns are reached at the end of 3 weeks of age. These findings indicate that parvalbumin and calbindin D28k-immunoreactivities in RSC neurons change during postnatal development. As for IL-18R, the present study showed that immunoreactivity for IL-18R changes in RSC neurons of the wild-type and IL-18KO mice during postnatal development. Because the IL-18R-ir neurons in layers II and V were different in their size and shape, it is likely that they do not migrate together. Adult patterns of IL-18R-ir neurons may reach at the 6 weeks of age different from those of parvalbumin and calbindin D28k-immunoreactive neurons. These differences may cause that parvalbumin and calbindin D28k-immunoreactive neurons play a role for interneurons in the cerebral cortex (De Felipe 1997), but IL-18R-ir neurons may not play important role for neuronal connections or circuits because no axon terminals express IL-18R immunoreactivity in the RSC.

When the sacral nerve or the trigeminal nerve is injured, a striking increase in IL-18 and IL-18R expression is observed in the glial cells but not in the neurons in the dorsal horn of the spinal cord or the trigeminal spinal nucleus caudalis (Miyoshi et al., 2008; Daigo et al., 2012). This led to the idea that injury of the trigeminal or sacral nerve induces IL-18 upregulation in glial cells in the sensory nuclei, activating sensory neurons for a possible role of IL-18 in neuropathic pain. These findings would then suggest that pain stimulation does not activate or influence directly to the neurons containing IL-18 or IL-18R. Whereas, IL-18R-ir neurons in layers II and III of RSC disappeared after 3 weeks of ages in both wild type and IL-18 KO mice. These results suggested that the construction of neural network may not relate appearance of IL-18R immunoreactivity in the RSC neurons.

Lesion studies targeting different part of the RSC, such as destruction by mechanical or pharmaceutical lesion, or alternation of projections to the hippocampus from the entorhinal cortex or the RSC in rats have shown a differential degree of an impairment for alterations in spatial memory performance and motor activity (Van Hoesen et al., 1991; Wyss and Van Groen, 1992; Wozniak et al., 1996; Vann et al., 2003; Vann and Aggleton, 2004; Sipos et al., 2007; Pothuizen et al., 2008). Behavioral studies have reported that the rearing activity is significantly suppressed, retention latency is much shorter, and the acquisition latency is significantly prolonged in the water maze test in IL-18KO mice compared with wild-type mice (Yaguchi et al., 2010; Too et al., 2014). These results suggest that behavioral abnormalities of IL-18KO mice may be caused by disappearance of IL-18R or degeneration of presumed IL-18 containing neurons in the RSC.

We have attempted to clarify when immunoreactivity for IL-18R disappears in layer V neurons in IL-18KO mice. Both the wild-type mice and the IL-18KO mice did not show IL-18R-ir neurons in layer V before 6 weeks of age, thus it is difficult to know when immunoreactivity for IL-18R disappeared or when the presumed IL-18R containing neurons degenerate in layer $\mathrm{V}$ of the IL-18KO mice. Since IL-18R-ir neurons appeared and disappeared in layers II and III from 2 to 3 weeks of age in both the wild-type and the IL-18KO mice, IL-18R immunoreactivity in layer $\mathrm{V}$ may develop around 3 weeks of age. The mammalian visual system is affected by altered visual experiences during a special developmental period known as the critical period from 2 to 3 weeks of ages (Wiesel and Hubel, 1963, 1965; Kawabata et al., 2003). Because mice open their eyes around 2 weeks of age and the RSC has reciprocal connections with the visual cortex (van Groen and Wyss, 1990, 1992, 2003; Shibata et al., 2004), indirect visual inputs may affect the IL-18R-containing neurons with or without IL-18 after 2 weeks of age. IL-18 has been detected in human epithelial cells of the lactating mammary gland by immunohistochemistry, human colostrum contains significantly higher levels of IL-18 compared with early milk and mature milk (Takahata et al., 2001). Further, the study showed a significantly correlation between levels of IL-18 in human milk and 
the occurrence of preterm delivery and pregnancy complications of mothers. Since the weaning period of mice is around 2 to 3 weeks of age, these behavioral changes may affect to the RSC neurons, which are involved behavioral activities such as head direction, spatial memory, navigation, or motor activity (Chen et al., 1994a, 1994b; Cooper and Mizumori, 1999; Harker and Whishaw, 2002). Thus, IL-18 or IL-18R seem to be involved in the construction of neural circuits corresponding to events after 3-weeks of ages. Further study will be needed to clarify what kind of effectors influence the immunoreactivity of IL-18R in the RSC neurons.

\section{Conflict of interest}

T. Hayakawa, M. Hata and H. Okamura disclose financial interests from Hirakata Ryoikuen related to this research to disclose.

\section{Acknowledgements}

We thank Ms. Fumiko Yoshida, Ms. Emi Yamaguchi, Ms. Naomi Gamachi and Ms. Atsuko Tamamoto for their technical support.

\section{References}

1) Alboni S, Cervia D, Ross B, Montanari C, Gonzalez AS, SanchezAlavez M, Marcondes MC, De Vries D, Sugama S, Brunello N, Blom J, Tascedda F, Conti B: Mapping of the full length and the truncated interleukin-18 receptor alpha in the mouse brain. Journal of neuroimmunology 2009; 214:43-54.

2) Alcantara S, Ferrer I, Soriano E: Postnatal development of parvalbumin and calbindin D28K immunoreactivities in the cerebral cortex of the rat. Anatomy and Embryology (Berlin) 1993; 188: 63-73.

3) Andoh T, Kishi H, Motoki K, Nakanishi K, Kuraishi Y, Muraguchi A: Protective effect of IL-18 on kainate- and IL-1 beta-induced cerebellar ataxia in mice. Journal of immunology 2008; 180:23222328.

4) Chen LL, Lin LH, Barnes CA, McNaughton BL: Head-direction cells in the rat posterior cortex. II. Contributions of visual and ideothetic information to the directional firing. Experimental brain research 1994a; 101:24-34.

5) Chen LL, Lin LH, Green EJ, Barnes CA, McNaughton BL: Headdirection cells in the rat posterior cortex. I. Anatomical distribution and behavioral modulation. Experimental brain research 1994b; 101:8-23.

6) Conti B, Sugama S, Kim Y, Tinti C, Kim H, Baker H, Volpe B, Attardi B, Joh T: Modulation of IL-18 production in the adrenal cortex following acute ACTH or chronic corticosterone treatment. Neuroimmunomodulation 2000; 8:1-7.

7) Cooper BG, Mizumori SJ: Retrosplenial cortex inactivation selectively impairs navigation in darkness. Neuroreport 1999; 10:625630.

8) Daigo E, Sakuma Y, Miyoshi K, Noguchi K, Kotani J: Increased expression of interleukin-18 in the trigeminal spinal subnucleus caudalis after inferior alveolar nerve injury in the rat. Neuroscience letters 2012; 529:39-44.

9) De Felipe J: Types of neurons, synaptic connections and chemical characteristics of cells immunoreactive for calbindin-D28K, parvalbumin and calretinin in the neocortex. Journal of chemical neuroanatomy 1997; 14:1-19.

10) Dinarello CA: Interleukin 1 and interleukin 18 as mediators of inflammation and the aging process. The American journal of clinical nutrition 2006; 83:447S-455S.

11) Dinarello CA: Interleukin- 18 and the pathogenesis of inflammatory diseases. Seminars in nephrology 2007; 27:98-114.

12) Dinarello CA, Fantuzzi G: Interleukin-18 and host defense against infection. The Journal of infectious diseases 2003; 187:Suppl 2 S370-384.

13) Harker KT, Whishaw IQ: Impaired spatial performance in rats with retrosplenial lesions: importance of the spatial problem and the rat strain in identifying lesion effects in a swimming pool. The Journal of neuroscience 2002; 22:1155-1164.

14) Hayakawa T, Hata M, Kuwahara-Otani $S$, Yamanishi K, Yagi H, Okamura H: Fine structure of interleukin 18 (IL-18) receptorimmunoreactive neurons in the retrosplenial cortex and its changes in IL18 knockout mice. Journal of chemical neuroanatomy 2016, 78:96-101.

15) Kawabata K, Maeda S, Takanaga A, Ito H, Tanaka K, Hayakawa T, Seki M: Apoptosis and retinal projections in the dorsal lateral geniculate nucleus after monocular deprivation during the later phase of the critical period in the rat. Anatomical Science International 2003; 78:104-110.

16) Kuwahara-Otani S, Maeda S, Kobayashi K, Minato Y, Tanaka K, Yamanishi K, Hata M, Li W, Hayakawa T, Noguchi K, Okamura $\mathrm{H}$, Yagi H: Interleukin-18 and its receptor are expressed in gonadotropin-releasing hormone neurons of mouse and rat forebrain. Neuroscience letters 2017; 650:33-37.

17) Luth HJ, Blumcke I, Winkelmann E, Celio MR: The calciumbinding protein calretinin is localized in a subset of interneurons in the rat cerebral cortex: a light and electron immunohistochemical study. Journal fur Hirnforschung 1993; 34:93-103.

18) Miyoshi $K$, Obata $K$, Kondo $T$, Okamura $H$, Noguchi $K$ : Interleukin-18-mediated microglia/astrocyte interaction in the spinal cord enhances neuropathic pain processing after nerve injury. The Journal of neuroscience 2008; 28:12775-12787.

19) Nagai $Y$, Nochi T, Watanabe $K$, Watanabe $K$, Aso H, Kitazawa H, Matsuzaki M, Ohwada S, Yamaguchi T: Localization of interleukin-18 and its receptor in somatotrophs of the bovine anterior pituitary gland. Cell and tissue research 2005; 322:455-462.

20) Okamura H, Tsutsi H, Komatsu T, Yutsudo M, Hakura A, Tanimoto T, Torigoe K, Okura T, Nukada Y, Hattori K, et al.: Cloning of a new cytokine that induces IFN-gamma production by $\mathrm{T}$ cells. Nature 1995; 378:88-91.

21) Pothuizen HH, Aggleton JP, Vann SD: Do rats with retrosplenial cortex lesions lack direction? The European journal of neuroscience 2008; 28:2486-2498.

22) Reddy P: Interleukin-18: recent advances. Current Opinion Hematology 2004; 11:405-410.

23) Salaj M, Druga R, Cerman J, Kubova H, Barinka F: Calretinin and parvalbumin immunoreactive interneurons in the retrosplenial cortex of the rat brain: Qualitative and quantitative analyses. Brain research 2015; 1627:201-215.

24) Shibata H, Kondo S, Naito J: Organization of retrosplenial cortical projections to the anterior cingulate, motor, and prefrontal cortices in the rat. Neuroscience research 2004; 49:1-11.

25) Sipos E, Kurunczi A, Kasza A, Horvath J, Felszeghy K, Laroche S, Toldi J, Parducz A, Penke B, Penke Z: Beta-amyloid pathology in the entorhinal cortex of rats induces memory deficits: implications for Alzheimer's disease. Neuroscience 2007; 147:28-36.

26) Sugama S, Cho BP, Baker H, Joh TH, Lucero J, Conti B: Neurons of the superior nucleus of the medial habenula and ependymal cells express IL-18 in rat CNS. Brain research 2002; 958:1-9. 
27) Takahata $Y$, Takada $H$, Nomura A, Ohshima $K$, Nakayama $H$, Tsuda T, Nakano H, Hara T: Interleukin-18 in human milk. Pediatric research 2001; 50:268-272.

28) Takeda K, Tsutsui $H$, Yoshimoto $T$, Adachi O, Yoshida $N$, Kishimoto T, Okamura H, Nakanishi K, Akira S: Defective NK cell activity and Th1 response in IL-18-deficient mice. Immunity 1998; 8:383-390.

29) Too LK, Mitchell AJ, Yau B, Ball HJ, McGregor IS, Hunt NH: Interleukin-18 deficiency and its long-term behavioural and cognitive impacts in a murine model of pneumococcal meningitis. Behavioural brain research 2014; 263:176-189.

30) van Groen T, Wyss JM: Connections of the retrosplenial granular a cortex in the rat. The Journal of comparative neurology 1990 ; 300:593-606.

31) van Groen T, Wyss JM: Connections of the retrosplenial dysgranular cortex in the rat. The Journal of comparative neurology 1992; 315:200-216.

32) Van Groen T, Wyss JM: Connections of the retrosplenial granular b cortex in the rat. The Journal of comparative neurology 2003 463:249-263.

33) Van Hoesen GW, Hyman BT, Damasio AR: Entorhinal cortex pathology in Alzheimer's disease. Hippocampus 1991; 1:1-8.

34) Vann SD, Aggleton JP: Testing the importance of the retrosplenial guidance system: effects of different sized retrosplenial cortex lesions on heading direction and spatial working memory. Behavioural brain research $2004 ; 155: 97-108$.
35) Vann SD, Kristina Wilton LA, Muir JL, Aggleton JP: Testing the importance of the caudal retrosplenial cortex for spatial memory in rats. Behavioural brain research 2003; 140:107-118

36) Wang N, Sugama S, Conti B, Teramoto A, Shibasaki T: Interleukin-18 mRNA expression in the rat pituitary gland. Journal of neuroimmunology 2006; 173:117-125.

37) Wiesel TN, Hubel DH: Effects of Visual Deprivation on Morphology and Physiology of Cells in the Cats Lateral Geniculate Body. Journal of neurophysiology 1963; 26:978-993.

38) Wiesel TN, Hubel DH: Comparison of the effects of unilateral and bilateral eye closure on cortical unit responses in kittens. Journal of neurophysiology 1965; 28:1029-1040.

39) Wozniak DF, Brosnan-Watters G, Nardi A, McEwen M, Corso TD, Olney JW, Fix AS: MK-801 neurotoxicity in male mice: histologic effects and chronic impairment in spatial learning. Brain research 1996; 707:165-179.

40) Wyss JM, Van Groen T: Connections between the retrosplenial cortex and the hippocampal formation in the rat: a review. Hippocampus 1992; 2:1-11.

41) Yaguchi $T$, Nagata $T$, Yang D, Nishizaki T: Interleukin-18 regulates motor activity, anxiety and spatial learning without affecting synaptic plasticity. Behavioural brain research 2010; 206:47-51.

42) Zgraggen E, Boitard M, Roman I, Kanemitsu M, Potter G, Salmon P, Vutskits L, Dayer AG, Kiss JZ: Early postnatal migration and development of layer II pyramidal neurons in the rodent cingulate/ retrosplenial cortex. Cerebral cortex 2012; 22:144-157. 